\title{
Educational needs of hematologists and laboratory professionals regarding factor activity assays
}

This article was published in the following Dove Press journal: Journal of Blood Medicine

\section{Dorothy M Adcock' \\ Mazi Rasulnia ${ }^{2}$ \\ Natalia Holot ${ }^{3}$ \\ David L Cooper ${ }^{3}$}

'Laboratory Corporation of America Holdings, Burlington, NC, ${ }^{2} \mathrm{M}$ Consulting LLC, Birmingham, AL, ${ }^{3}$ Clinical, Medical, and Regulatory Affairs, Novo Nordisk Inc., Plainsboro, NJ, USA
Correspondence: Dorothy M Adcock Laboratory Corporation of America Holdings, 53I South Spring Street, Burlington, NC 27215, USA $\mathrm{Tel}+\mathrm{I} 3364367716$

Email adcockd@labcorp.com
Introduction: Diagnosis and management of hemophilia require accurate and precise measurements of factor activity levels. Activity is traditionally measured via one-stage (OS) clot-based assay; however, chromogenic substrate (CS) assays may be needed for certain cases. A survey was performed to understand assay-related knowledge gaps among hematologists and laboratory professionals.

Methods: Separate web-based surveys were administered to hematologists who manage hemophilia and to laboratory professionals and queried practice patterns, knowledge of/attitudes toward CS assays, and interest in continuing education.

Results: A total of 51 hematologists participated in this study; 67\% managed hemophilia patients for $\geq 10$ years and $24 \%$ were affiliated with a hemophilia treatment center (HTC). Most $(80 \%)$ stated familiarity with general assay interpretation. Majorities of non-HTC and HTC respondents agreed that CS assays are more accurate than OS assays (62\%/67\%), although non-HTC hematologists indicated less understanding of when to order a CS assay $(49 \% / 67 \%)$. Fewer non-HTC respondents expressed concerns regarding the reliability of OS assays for diagnosis (38\%/67\%) and monitoring (38\%/75\%). Most (80\%) expressed an interest in factor assay education, especially on available assays, efficacy, and best practices (39\%). A total of 57 laboratory professionals participated, averaging 10 years in their current position; most $(88 \%)$ were hospital based. More performed OS (72\%) than CS (10\%) or both (17\%) assays; only $11 \%$ reported confidence with the interpretation of CS results. Few expressed concerns regarding the reliability of OS for diagnosis (9\%) or monitoring (12\%). Reported barriers to CS use included infrequent need (68\%), lack of US Food and Drug Administration (FDA) approval (61\%), and need for validation work (56\%). Most (70\%) were interested in CS assay education; top interests included advantages over traditional assays, general information on CS assays, and indications for testing (each 18\%).

Conclusion: Future educational efforts may focus on limitations of OS assays, indications for CS assay diagnosis/monitoring, and support for clinic-laboratory dialog.

Keywords: hemophilia, factor activity assay, chromogenic substrate assay, coagulation factor VIII, coagulation factor IX, monitoring

\section{Introduction}

Hemophilia $\mathrm{A}$ and $\mathrm{B}$ are bleeding disorders characterized by deficiencies in clotting factor VIII (FVIII) and factor IX (FIX), respectively. Accurate and precise measurements of patients' plasma factor activity levels are necessary for the diagnosis and classification of hemophilia with severe, moderate, and mild disease defined by activity levels of $<1,1$ to $5 \%$, and $>5$ to $<40 \%$ of normal plasma levels, respectively. ${ }^{1}$ Additionally, 
accurate and precise factor activity assessments are critical in determining effective doses for surgical, on-demand, and prophylactic factor replacement therapy, as dosing regimens are typically based on desired increases in FVIII/FIX levels or influenced by personalized pharmacokinetic parameters. ${ }^{2}$

Factor activity is traditionally measured using a one-stage (OS) clot-based assay, which relies on the assessment of activated partial thromboplastin time (aPTT). ${ }^{3}$ As aPTT is prolonged in individuals with hemophilia A or B, the OS assay measures the degree of aPTT correction in factor-deficient plasma as diluted test plasma is added, to derive a measurement of test plasma factor level. Important advantages of this assay include a wide availability of testing reagents, large measurement range, and extensive accumulated experience with its use. However, substantial variability exists when comparing results between laboratories using different OS assays, due to issues including the wide variety of aPTT reagents and different types of activators, sources of factordeficient plasma, variations in assay methodology, and clot detection properties of different automated analyzers. ${ }^{3-5}$

The chromogenic substrate (CS) assay is a two-stage assay designed to overcome many of the limitations inherent in OS assays. The first stage of this test involves incubation of diluted test plasma to generate activated factor X (FXa), under conditions in which FVIII activity (CS-FVIII assay) or FIX activity (CS-FIX assay) is rate limiting. The amount of FXa generated is then measured indirectly as production of a chromogenic signal via FXa-induced cleavage of a chromogen-peptide substrate. Although infrequently used in the United States due to issues such as cost, lack of familiarity, and for FIX a lack of US Food and Drug Administration (FDA) approval, CS assays offer improved accuracy, potentially a wider range of sensitivity, and less assay interference compared with OS assays. ${ }^{3,6}$ Discrepancies between OS and CS assays have been well documented and appear to be particularly problematic for diagnosing nonsevere hemophilia and for monitoring individuals prescribed newer, modified factor products. ${ }^{3,6-9}$ For example, use of OS assays may overestimate or underestimate FVIII levels in as many as $40 \%$ of patients with nonsevere hemophilia A, potentially resulting in a missed diagnosis or inaccurate classification of severity. ${ }^{6,10}$ For these reasons, laboratories routinely performing factor assays are recommended to strongly consider the addition of FVIII and possibly FIX CS assays. ${ }^{11}$

Knowledge of the limitations inherent in the use of OS assays and the potential benefits of CS assays is critical in ensuring that tests are performed and interpreted consistently and accurately. This needs assessment survey was performed to understand current knowledge gaps and learning needs of hematologists and laboratory professionals who oversee factor activity assay testing and aimed to identify opportunities to increase the awareness of how existing assays may be optimized for accurate and precise factor activity measurements.

\section{Methods}

Two distinct online surveys were fielded: one survey was completed by adult and pediatric hematologists and one survey was completed by laboratory professionals. The study was exempt from institutional review board approval, and informed consent was not required, as no private or protected information was obtained, no patients were surveyed, and no specific patient cases were referenced. All participants were provided a stipend of $\$ 150$ as compensation for their time.

\section{Hematologist survey}

The hematologist survey was fielded three times between March 14 and March 22, 2016, to a total of 3654 hematologists. Respondents were required to meet the following two inclusion criteria: to have a self-identified specialty of "adult hematology", "adult hematology with a focus on hemostasis and thrombosis", "adult hematology/oncology", "pediatric hematology/oncology", or "pediatric hematology with a focus on hemostasis and thrombosis" and to currently be managing patients with hemophilia. The survey included 16 questions, which assessed participant demographics, practice patterns surrounding the use of CS and OS assays, knowledge of CS assays, awareness of and attitudes toward the use of chromogenic factor assays, barriers to the use of CS-FVIII assays, and interest in continuing education topics.

\section{Laboratory professional survey}

The laboratory professional survey was fielded three times between November 30, 2015, and January 8, 2016, to a total of 1496 members of the Clinical Laboratory Management Association (CLMA) who opted in to receive email communications. The survey included 23 questions, which assessed practice demographics, practice patterns surrounding the use of FVIII and FIX assays, knowledge of CS-FVIII assays, awareness of and attitudes toward the use of CS factor assays, barriers to the use of CS-FVIII assays, and interest in continuing education topics and delivery format preferences.

\section{Analysis}

Most survey questions used 5- or 10-point response scales in which responses were grouped within three domains 
(eg, disagree/neutral/agree or not significant/somewhat significant/significant) and are reported as grouped categories. Data are reported via descriptive statistics (frequency counts, percentage, or mean).

\section{Results}

\section{Hematologist survey}

The hematologist survey was completed by a total of 51 adult and pediatric hematologists. Of these, 12 (24\%) were affiliated with a hemophilia treatment center (HTC) and 86\% were specialists in adult hematology. Most hematologists worked in an urban (57\%) or suburban (31\%) setting, in a group practice $(55 \%)$ or medical school $(23 \%)$, and in directed patient care activities (98\%). Majorities of both HTC-affiliated and non-HTC-affiliated hematologists reported having managed patients with hemophilia for $>10$ years (Table 1). However, most HTC-affiliated hematologists reported seeing $>10$ patients with hemophilia each week (84\%) and most nonHTC-affiliated hematologists reported seeing $\leq 10(87 \%)$ patients. Approximately half $(52 \%)$ of the hematologists reported ordering $>10$ factor assays each month; in general, non-HTC-affiliated hematologists reported ordering fewer assays.

Overall, the most common circumstance for which hematologists ordered factor activity testing was initial diagnosis (Figure 1). HTC-affiliated hematologists were more likely than non-HTC-affiliated hematologists to order tests at regular intervals (58 vs 31\%) and when patients required on-demand factor replacement (58 vs 41\%). Compared with HTC-affiliated hematologists, non-HTC-affiliated hematologists reported using CS assays less frequently to assist in diagnosis (46 vs 75\%) and monitoring (33 vs 75\%). Approximately one-third of all respondents indicated that they did not have ready access to a CS assay at their institution (HTC-affiliated hematologists, 50\%; non-HTC-affiliated hematologists, 31\%).

Among all hematologists, most (80\%) indicated familiarity with general assay interpretation. Non-HTC-affiliated hematologists were less familiar than HTC-affiliated hematologists with how coagulation laboratories operate (56 vs $75 \%$ ) and were approximately half as likely to have had previous training in a coagulation laboratory (36 vs $75 \%$ ) (Figure 2). Although the majority of both non-HTC-affiliated and HTC-affiliated hematologists agreed with the statement that CS assays are more accurate than OS assays (62 and 67\%, respectively), non-HTC-affiliated hematologists indicated less understanding of when to order a CS assay (49 vs 67\%) (Figure 3A). A smaller proportion of non-HTC-affiliated vs HTC-affiliated hematologists expressed concerns regarding the reliability of OS assays for both diagnosis (38 vs 67\%) and monitoring (38 vs 75\%) (Figure 3B).

Most hematologists expressed an interest in factor assay education (80\%). Specifically, top areas of interest included available assays, efficacy, and best practices (39\%), novel assays (15\%), new and available treatments (15\%), and assay quality $(12 \%)$. When queried regarding the most influential

Table I Hematologist practice characteristics.

\begin{tabular}{|c|c|c|c|}
\hline Characteristic & $\begin{array}{l}\text { Percentage of total } \\
\text { hematologists }(\mathrm{N}=5 \mathrm{I})\end{array}$ & $\begin{array}{l}\text { Percentage of HTC-affiliated } \\
\text { hematologists }(n=\mid 2)\end{array}$ & $\begin{array}{l}\text { Percentage of non-HTC- } \\
\text { affiliated hematologists }(n=39)\end{array}$ \\
\hline \multicolumn{4}{|l|}{ Years in practice } \\
\hline $1-5$ & 13 & 0 & 18 \\
\hline $6-10$ & 20 & 25 & 18 \\
\hline $\mathrm{II}-20$ & 43 & 58 & 38 \\
\hline $21-30$ & 18 & 17 & 18 \\
\hline$>30$ & 6 & 0 & 8 \\
\hline \multicolumn{4}{|c|}{ Number of patients seen each week } \\
\hline$\leq 1$ & 23 & 8 & 28 \\
\hline $2-10$ & 47 & 8 & 59 \\
\hline $11-20$ & 12 & 34 & 5 \\
\hline $21-30$ & 4 & 8 & 3 \\
\hline$>30$ & 14 & 42 & 5 \\
\hline \multicolumn{4}{|c|}{ Number of factor assays ordered each month } \\
\hline$\leq 1$ & 16 & 0 & 21 \\
\hline $2-10$ & 32 & 8 & 39 \\
\hline $11-20$ & 22 & 33 & 18 \\
\hline $21-30$ & 22 & 33 & 18 \\
\hline$>30$ & 8 & 25 & 3 \\
\hline
\end{tabular}

Abbreviation: HTC, hemophilia treatment center. 

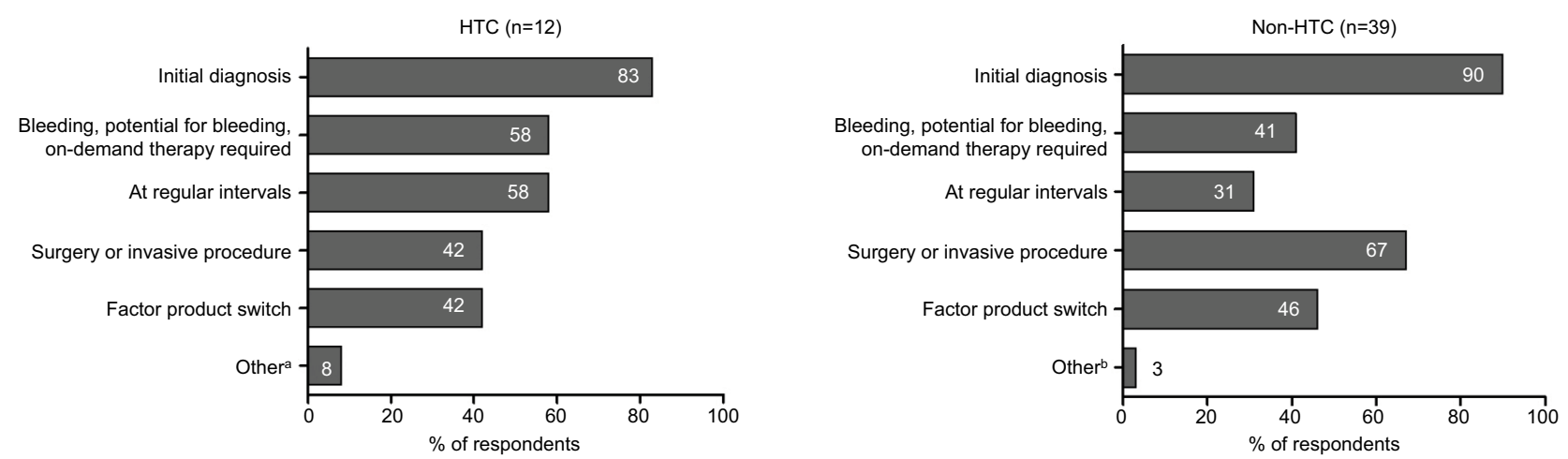

Figure I Hematologists' circumstances for ordering factor activity testing.

Notes: Participants selected all options that applied. ${ }^{a}$ Other was specified as change in bleeding phenotype. ${ }^{b}$ Other was specified as central nervous system bleeding. Abbreviation: HTC, hemophilia treatment center.

$\operatorname{HTC}(n=12)$

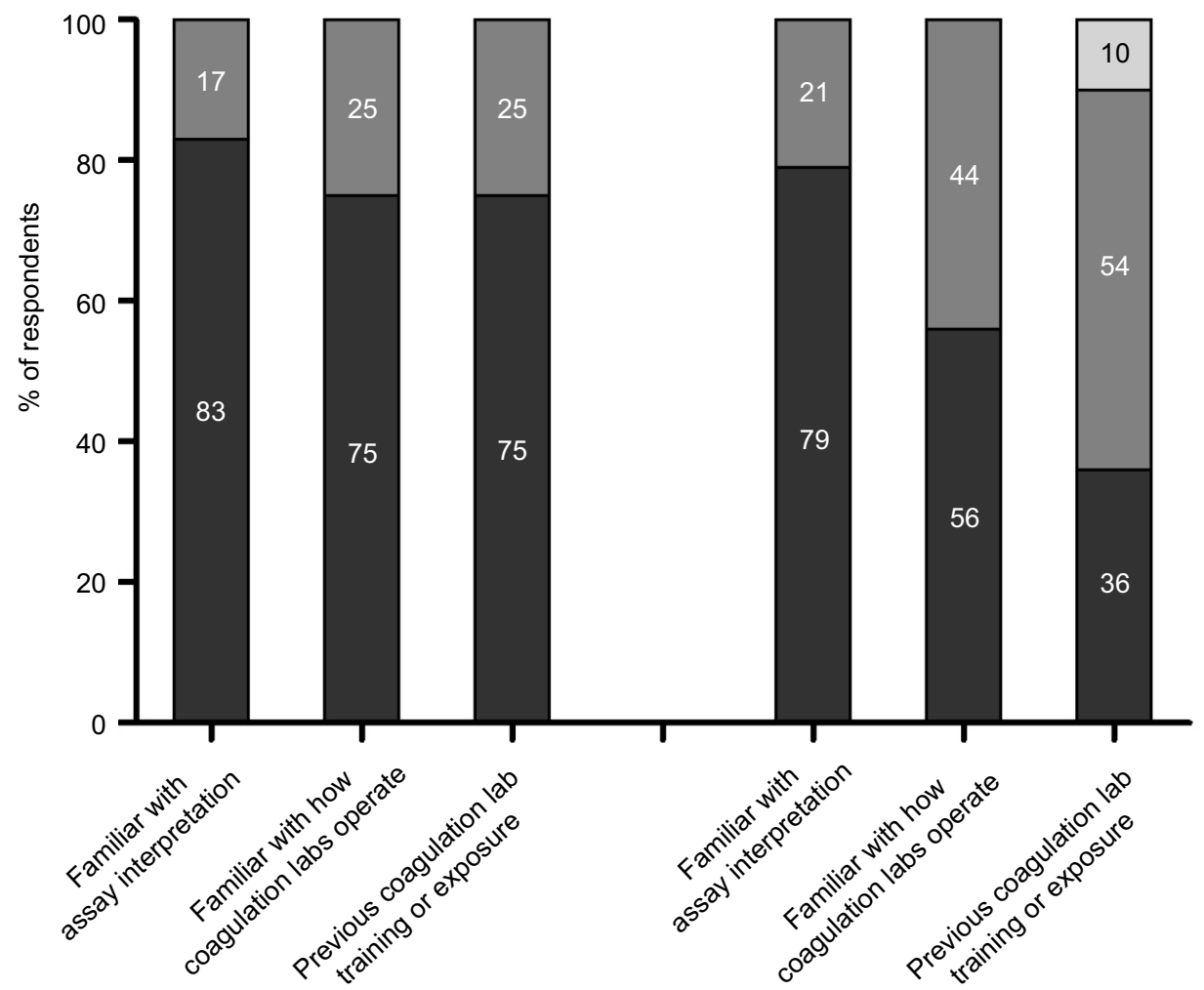

Non-HTC $(n=39)$

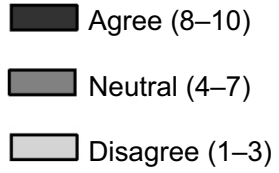

Figure $\mathbf{2}$ Hematologists' general familiarity with coagulation assays and laboratories. Abbreviation: HTC, hemophilia treatment center.

sources of information for adopting new practices, the most common responses included clinical guidelines (84\%), peerreviewed journal articles (84\%), national experts (74\%), and colleagues and peers $(69 \%)$.

\section{Laboratory professional survey}

The laboratory professional survey was completed by a total of 57 individuals, including laboratory directors (35\%), managers (28\%), and supervisors/coordinators (14\%) (Table 2). Most respondents had been in their current position for several years, with a mean duration of 10 years. Most (88\%) worked in a hospital-based laboratory, and only $18 \%$ were affiliated with an HTC. Nearly all (94\%) reported having input into their laboratory's assay selection. A total of $36 \%$ reported directly performing $>20$ FVIII assays each year (overall 23\% for diagnosis and 24\% for monitoring purposes) 

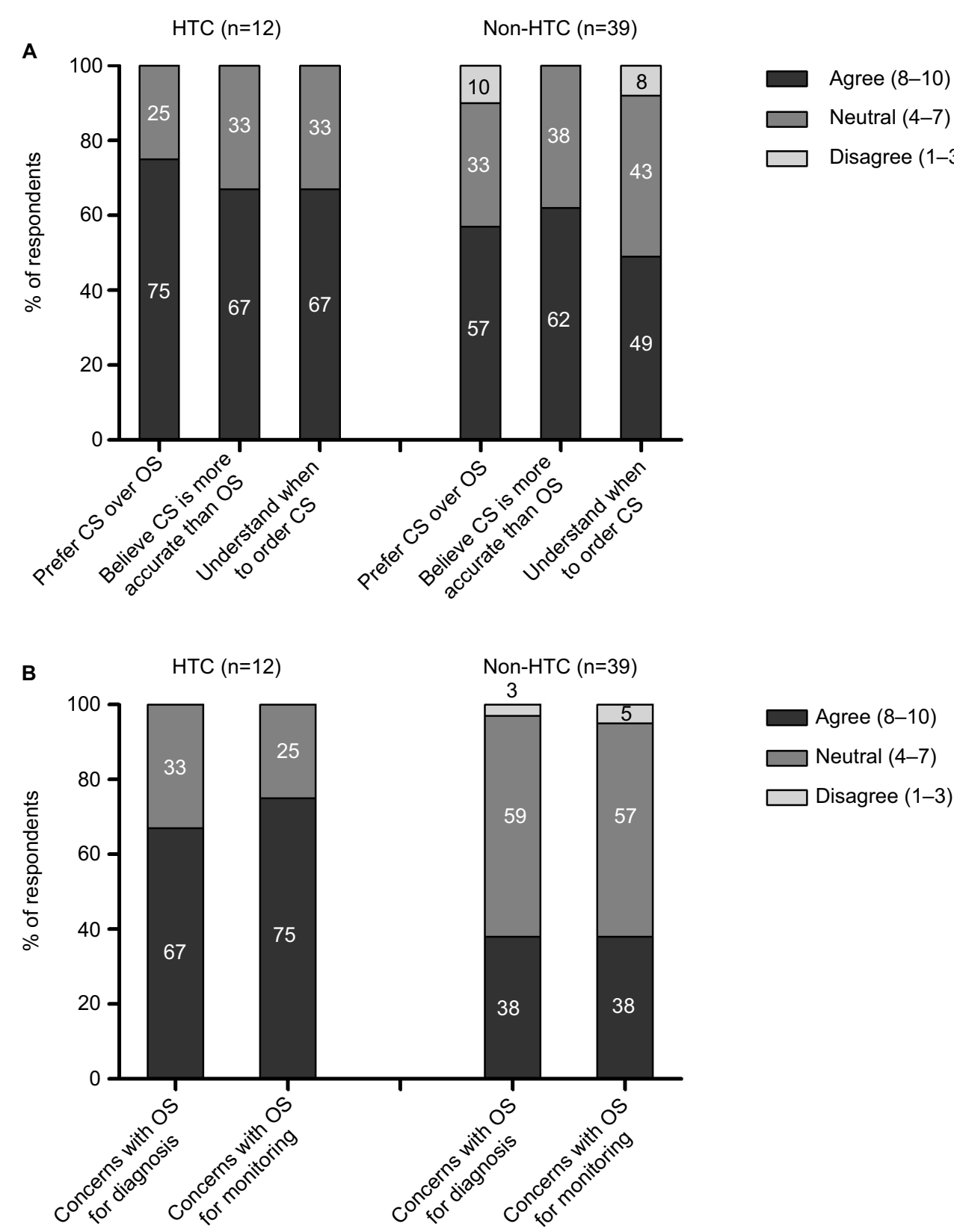

Figure 3 Hematologists' perceptions regarding chromogenic assays (A) and concerns regarding OS assays (B). Abbreviations: CS, chromogenic substrate; HTC, hemophilia treatment center; OS, one stage.

and $16 \%$ reported directly performing $>20$ FIX assays each year (overall $16 \%$ for diagnosis and $12 \%$ for monitoring purposes). When measuring FVIII levels for diagnosis and monitoring purposes, more laboratory professionals reported performing OS assays (72\%) than CS assays (10\%) or both types of assays (17\%). A limited number of laboratory professionals reported having CS-FVIII and CS-FIX assays available in their laboratories (5 and 2\%, respectively).

Nearly half (44\%) of the laboratory professionals reported having no familiarity with CS assays; only $26 \%$ were "very" or "somewhat" familiar with these assays. Reported confidence with the interpretation of CS assay results was relatively low (11\%); additionally, only $11 \%$ of laboratory professionals reported being familiar with the advantages of a CS assay and $19 \%$ agreed with the statement that CS assays are more accurate than OS assays (Figure 4A). Only a small minority of participants (4\%) agreed that CS assays should be recommended for the evaluation of mild hemophilia. Additionally, few respondents expressed concerns regarding the reliability of OS assays for both diagnosis (9\%) and monitoring (12\%) (Figure 4B). When queried regarding barriers to CS assay use, laboratory professionals most often cited 
Table 2 Laboratory professional characteristics

\begin{tabular}{|c|c|}
\hline Characteristic & $\begin{array}{l}\text { Percentage of laboratory } \\
\text { professionals }(\mathrm{N}=57)\end{array}$ \\
\hline \multicolumn{2}{|l|}{ Professional title } \\
\hline Director & 35 \\
\hline Manager & 28 \\
\hline Supervisor/coordinator & 14 \\
\hline Technician/scientist & 9 \\
\hline Administrator & 5 \\
\hline Others & 7 \\
\hline \multicolumn{2}{|c|}{ Number of years in current position } \\
\hline$\leq 4$ & 32 \\
\hline $5-9$ & 25 \\
\hline $10-14$ & 19 \\
\hline$\geq 15$ & 25 \\
\hline \multicolumn{2}{|l|}{ Organization type } \\
\hline Hospital & 88 \\
\hline Independent laboratory & 11 \\
\hline Government laboratory & 2 \\
\hline \multicolumn{2}{|l|}{ HTC affiliation } \\
\hline Affiliated & 18 \\
\hline Not affiliated & 77 \\
\hline Not sure & 5 \\
\hline \multicolumn{2}{|c|}{ Number of FVIII assays directly } \\
\hline \multicolumn{2}{|c|}{ performed annually } \\
\hline 0 & 39 \\
\hline $1-20$ & 25 \\
\hline $21-100$ & 16 \\
\hline $10 \mid-500$ & 10 \\
\hline$>500$ & 10 \\
\hline \multicolumn{2}{|l|}{ Number of FIX assays directly } \\
\hline \multicolumn{2}{|l|}{ performed annually } \\
\hline 0 & 49 \\
\hline $\mathrm{I}-20$ & 35 \\
\hline $21-100$ & 4 \\
\hline $101-500$ & 8 \\
\hline$>500$ & 4 \\
\hline \multicolumn{2}{|c|}{ Assay use for diagnosis and monitoring } \\
\hline \multicolumn{2}{|l|}{ to measure FVIII } \\
\hline Chromogenic assay & 10 \\
\hline \multicolumn{2}{|c|}{ Both one-stage and chromogenic } \\
\hline assays & 17 \\
\hline
\end{tabular}

Abbreviations: FVIII, factor VIII; FIX, factor IX; HTC, hemophilia treatment center.

infrequent need (68\%), lack of FDA approval (61\%), need for additional validation work (56\%), lack of technology or expertise (54\%), and cost (49\%) (Figure 4C).

The most common informational resources cited by laboratory professionals for adopting new practices were clinical guidelines ( $92 \%)$, colleagues and peers $(64 \%)$, peer-reviewed journals (62\%), and national experts (58\%). Common websites visited for medical information included the College of American Pathologists (CAP) Today (24\%) and those of the American Association for Clinical Chemistry (AACC, $18 \%)$ and CLMA (18\%). Frequently read professional journals included the Medical Laboratory Observer (22\%), CAP Today (20\%), and ADVANCE for Medical Laboratory Professionals (6\%). The most frequently attended conferences were those of the CLMA (62\%), AACC (26\%), and American Society for Clinical Pathology (16\%).

Most laboratory professionals reported being interested in CS assay education (70\%), with top areas of interest including advantages over OS assays (18\%), general information on CS assays (18\%), indications for testing (18\%), and testing validation and quality control (11\%). Preferred formats for medical education indicated by respondents included live online meetings (84\%), local meetings (within 1 hour of individuals' practice location; 76\%), peer-reviewed journals (65\%), online courses (65\%), and websites (65\%) (Figure 5).

\section{Discussion}

The data identify important educational needs of both hematologists and laboratory professionals in the United States regarding the use and interpretation of FVIII and FIX activity assays. Both survey populations reflect a considerable level of experience, with most hematologists reporting $>10$ years in practice, and nearly half of the laboratory professionals reporting having been employed in their current position for at least 10 years. However, analysis of participant knowledge, attitudes toward, and familiarity with different types of assays suggests specific opportunities to improve the awareness of assay limitations and to improve the accuracy, precision, and consistency of test results.

Most hematologists surveyed in this study reported being familiar with assay interpretation, although fewer were familiar with the details of coagulation laboratory testing. In particular, hematologists who were not affiliated with an HTC appeared to have the lowest levels of specific knowledge regarding testing issues, as nearly half of the non-HTC-affiliated hematologists reported being unfamiliar with how coagulation laboratories operate and approximately two-thirds lacked previous training in a coagulation laboratory. Non-HTC-affiliated hematologists may also benefit from greater awareness of patient circumstances warranting factor activity testing, as substantially fewer of these participants compared with those affiliated with an HTC reported ordering assays at regular intervals or in response to the need for on-demand therapy, which may be the important circumstances for individualizing therapy based on personalized pharmacokinetics. ${ }^{12,13}$

Greater knowledge regarding the limitations of OS assays and potential benefits of CS assays may be an important aspect of education for all hematologists. Approximately half of the 

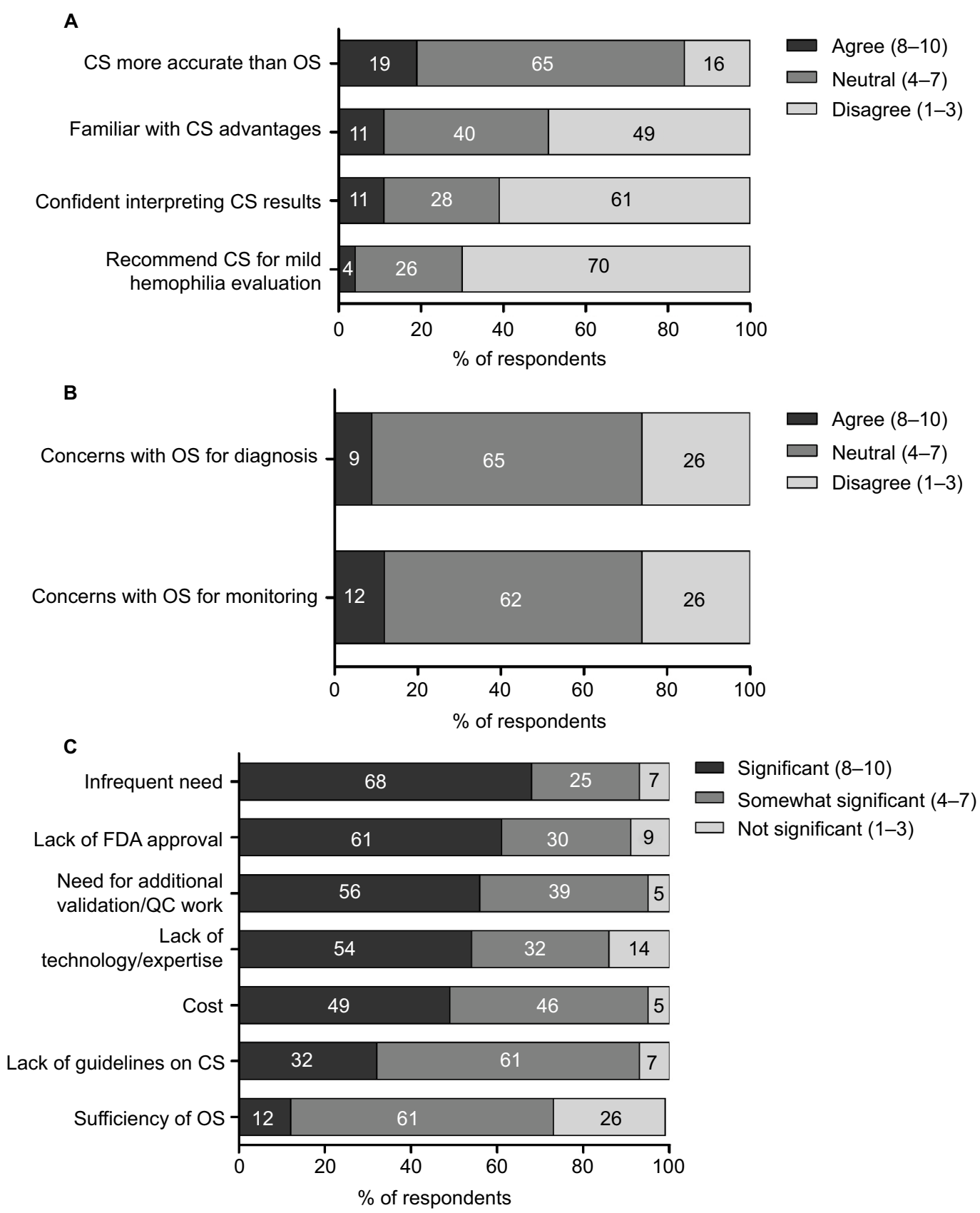

Figure 4 Laboratory professionals' perceptions regarding chromogenic assays $(\mathbf{A})$, concerns regarding OS assays (B), and perceived barriers to chromogenic assay use $(\mathbf{C})$. Abbreviations: CS, chromogenic substrate; FDA, US Food and Drug Administration; OS, one stage; QC, quality control.

hematologists reported understanding when to order a CS assay, and similarly, few reported having any concerns with the use of OS assays for diagnostic or monitoring purposes. When compared with hematologists who worked with an HTC, those unaffiliated with an HTC reported less frequent use of CS assays and less concern about the reliability of OS assays, suggesting important knowledge gaps within this population related to the advantages of CS assay use. Assay issues have been apparent in the past, even with standard recombinant FVIII products ${ }^{14}$ and with single-chain recombi- nant FVIII requiring doubling the OS results. ${ }^{15}$ However, the lack of awareness of OS assay limitations may become more problematic in the context of greater use of newer, modified (standard and extended-half-life) FVIII and FIX products, as careful selection of reagents and methods may be necessary to avoid overestimation or underestimation of factor activity levels, and some OS assays are effectively unsuitable for monitoring certain extended half-life products due to extreme estimation errors. ${ }^{3}$ Additionally, the limited accessibility of CS assays reported by hematologists may contribute to the 


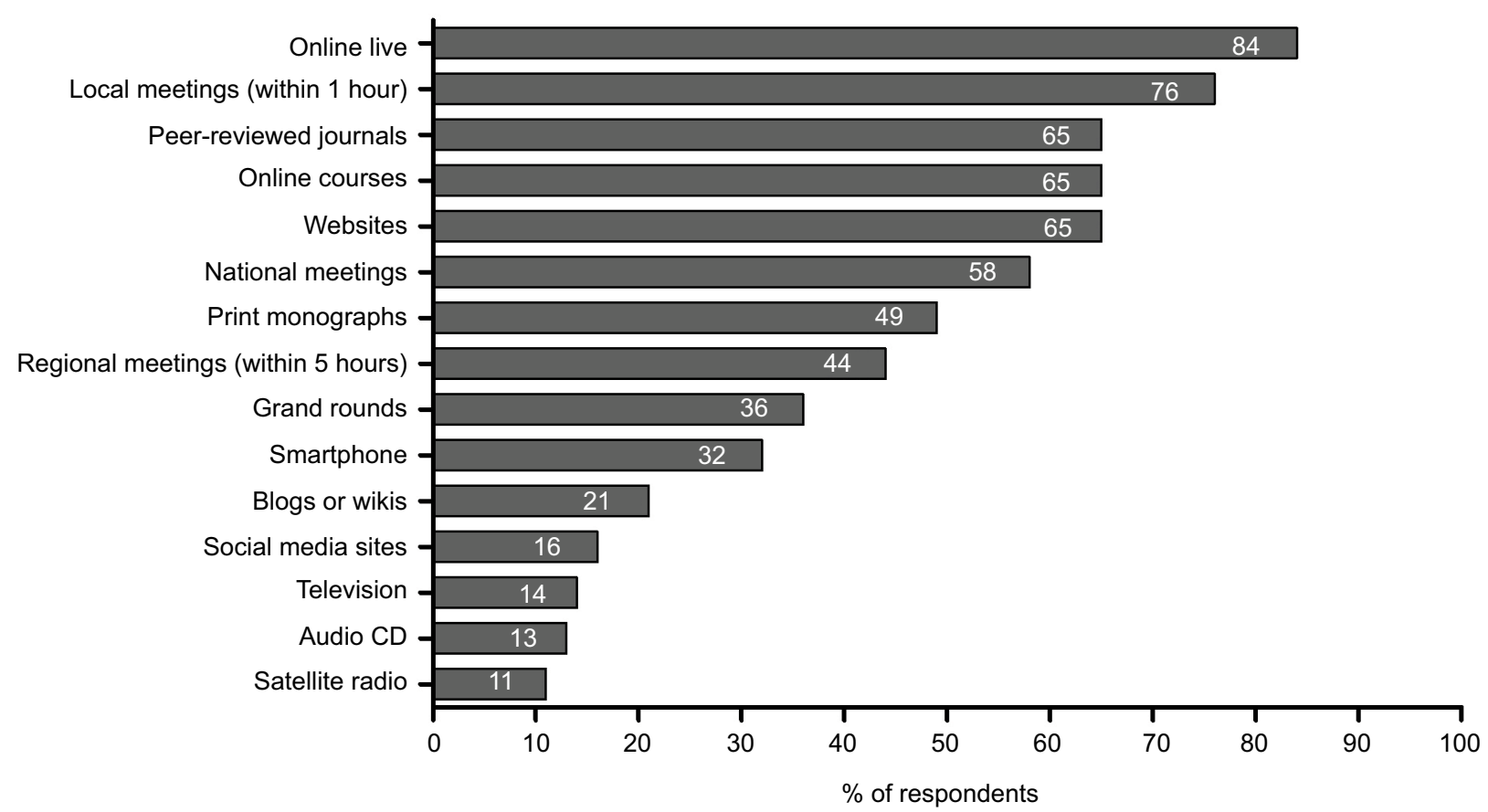

Figure 5 Laboratory professionals' preferred formats for receiving medical education.

limited understanding of their use and may constitute a barrier toward greater adoption of this assay technique.

Similar to the hematologist participants, laboratory professionals reported substantial experience with OS-FVIII/ FIX testing for the diagnosis and monitoring of hemophilia, but limited familiarity with performing and interpreting CSFVIII and CS-FIX assays. Most reported little institutional availability of CS assays and were not familiar with or confident in how CS assays could be used to augment diagnosis and monitoring of hemophilia. Laboratory professionals also appeared to be less aware of OS assay limitations than hematologists, as fewer of these participants agreed that CS assays are more accurate than OS assays or expressed concerns with the use of OS assays for patient diagnosis or monitoring. Additionally, only a small minority of laboratory professionals agreed that CS assays should be recommended in cases of nonsevere hemophilia (ie, circumstances in which OS assay results are likely to overestimate or underestimate FVIII levels), despite professional guidance that laboratories should strongly consider the addition of FDA-approved CS-FVIII and CS-FIX assays. ${ }^{11}$ Of note, obtaining FDA approval of CS-FIX assays may be an important means of increasing their use, as a lack of approved assays was indicated by a majority of laboratory professionals as a significant barrier toward greater CS assay use; however, it should be mentioned that multiple CS-FVIII assays have already been FDA approved. Additional barriers that were commonly indicated included the need for validation work and the lack of technology or expertise, suggesting specific needs for education in these areas. Furthermore, approximately half of the laboratory professionals mentioned cost as a significant barrier to CS assay use, suggesting that efforts aimed at reducing costs may help to promote greater use of this technique. Additionally, cost may reflect the underlying research use of CS assays in clinical trials, including large volume kits that may be less appropriate for smaller laboratories with lower or more sporadic test volume.

Majorities of both hematologists and laboratory professionals indicated an interest in factor assay education, suggesting that these audiences would be receptive to educational offerings. For hematologists, important information may include illustrative cases of when to use CS factor activity assays for diagnosis and monitoring of hemophilia, to increase confidence among clinicians and to improve patient care. For laboratory professionals, valuable efforts may include continuing education programs (eg, contextual cases to illustrate the use of CS-FVIII/FIX assays) aimed at increasing individuals' confidence with recommending and implementing broader OS/CS approaches in local laboratories or networks.

Key limitations of this study include the elective nature of enrollment and limited sample sizes. Individuals with an interest in factor assays may be expected to have been most likely to respond; additionally, the laboratory professional survey was fielded only to CLMA members who opted in 
to receive email communications, which may further bias the study population to individuals with the highest level of engagement in the clinical laboratory management community. However, overall findings are largely consistent with separate global surveys of hemophilia physicians and laboratory scientists, which indicated low rates of CS assay use and potential opportunities to improve the understanding of assay types and capabilities. ${ }^{4,5}$ Additionally, well-documented limitations of OS assays, particularly in the context of the diagnosis and classification of nonsevere hemophilia, as well as the expanding use of modified (standard and extended half-life) FVIII and FIX products ensure that careful consideration of specific testing requirements and capabilities will be critical in improving the accuracy and consistency of assay results necessary for high-quality patient care. ${ }^{3,6,8-10}$

\section{Acknowledgments}

Writing assistance was provided by Anna Abt, PhD, of ETHOS Health Communications in Yardley, PA, USA, and was supported financially by Novo Nordisk Inc., Plainsboro, NJ, USA, in compliance with international Good Publication Practice guidelines. Abstracts of this study were presented at the 10th Scientific Symposium of the Hemostasis and Thrombosis Research Society (HTRS) and the 30th International Symposium on Technical Innovations in Laboratory Hematology (ISLH), as poster presentations. The HTRS abstract is published in Haemophilia: http://onlinelibrary.wiley.com/doi/10.1111/ hae.2017.23.issue-S3/issuetoc, and the ISLH abstract is published in the International Journal of Laboratory Hematology: http://onlinelibrary.wiley.com/doi/10.1111/ijlh.2017.39.issueS2/issuetoc. This research was sponsored by Novo Nordisk Inc.

\section{Disclosure}

NH and DLC are employees of Novo Nordisk Inc. DMA has served as a consultant for Novo Nordisk and Baxalta. MR received a contract to execute the survey. The authors report no other conflicts of interest in this work.

\section{References}

1. Blanchette VS, Key NS, Ljung LR, et al. Definitions in hemophilia: communication from the SSC of the ISTH. J Thromb Haemost. 2014;12(11):1935-1939.

2. Srivastava A, Brewer AK, Mauser-Bunschoten EP, et al. Guidelines for the management of hemophilia. Haemophilia. 2013;19(1):e1-e47.

3. Kitchen S, Kershaw G, Tiefenbacher S. Recombinant to modified factor VIII and factor IX - chromogenic and one-stage assays issues. Haemophilia. 2016;22(suppl 5):72-77.

4. Gomez K, Chitlur M; GEHEP Panel. Survey of laboratory tests used in the diagnosis and evaluation of haemophilia A. Thromb Haemost. 2013;109(4):738-743.

5. Kitchen S, Signer-Romero K, Key NS. Current laboratory practices in the diagnosis and management of haemophilia: a global assessment. Haemophilia. 2015;21(4):550-557.

6. Potgieter JJ, Damgaard M, Hillarp A. One-stage vs. chromogenic assays in haemophilia A. Eur J Haematol. 2015;94(suppl 77):38-44.

7. Dodt J, Hubbard AR, Wicks SJ, et al. Potency determination of factor VIII and factor IX for new product labelling and postinfusion testing: challenges for caregivers and regulators. Haemophilia. 2015;21(4) 543-549.

8. Armstrong E, Hillarp A. Assay discrepancy in mild haemophilia A. Eur J Haematol Suppl. 2014;76:48-50.

9. Wilmot HV, Hogwood J, Gray E. Recombinant factor IX: discrepancies between one-stage clotting and chromogenic assays. Haemophilia. 2014;20(6):891-897.

10. Gilmore R, Harmon S, Gannon C, Byrne M, O'Donnell JS, Jenkins PV. Thrombin generation in haemophilia A patients with mutations causing factor VIII assay discrepancy. Haemophilia. 2010;16(4):671-674.

11. National Hemophilia Foundation. MASAC Document \#228: MASAC Statement Regarding Use of Various Clotting Factor Assays to Monitor Factor Replacement Therapy; 2014. Available from: https://www.hemophilia.org/sites/default/files/document/files/masac-228.pdf. Accessed February 24, 2018.

12. Petrini P, Valentino LA, Gringeri A, Re WM, Ewenstein B. Individualizing prophylaxis in hemophilia: a review. Expert Rev Hematol. 2015;8(2):237-246.

13. Pruthi RK. Laboratory monitoring of new hemostatic agents for hemophilia. Semin Hematol. 2016;53(1):28-34.

14. ReFacto [prescribing information]. Philadelphia, PA: Wyeth Pharmaceuticals; 2007. Available from: http://labeling.pfizer.com/showlabeling. aspx?id=140. Accessed March 14, 2018.

15. Afstyla [prescribing information]. Marburg, Germany: CSL Behring; 2017. Available from: http://labeling.cslbehring.com/PI/US/Afstyla/ EN/Afstyla-Prescribing-Information.pdf. Accessed March 14, 2018.
Journal of Blood Medicine

Publish your work in this journal

The Journal of Blood Medicine is an international, peer-reviewed, open access, online journal publishing laboratory, experimental and clinical aspects of all aspect pertaining to blood based medicine including but not limited to: Transfusion Medicine; Blood collection, Donor issues, Transmittable diseases, and Blood banking logistics; Immunohematology; Artificial and alternative
Dovepress

blood based therapeutics; Hematology; Biotechnology/nanotechnology of blood related medicine; Legal aspects of blood medicine; Historical perspectives. The manuscript management system is completely online and includes a very quick and fair peer-review system. Visit http://www.dovepress.com/ testimonials.php to read real quotes from published authors. 